\title{
Architected Lattices for Simultaneous Broadband Attenuation of Airborne Sound and Mechanical Vibrations in All Directions
}

\author{
Osama R. Bilal, ${ }^{1,2,{ }^{*}}$ David Ballagi, ${ }^{1}$ and Chiara Daraio ${ }^{2}$ \\ ${ }^{1}$ Department of Mechanical and Process Engineering, ETH Zurich, 8092 Zurich, Switzerland \\ ${ }^{2}$ Division of Engineering and Applied Science, California Institute of Technology, Pasadena, \\ California 91125, USA
}

(Received 31 August 2018; published 27 November 2018)

\begin{abstract}
Phononic crystals and acoustic metamaterials are architected lattices designed to control the propagation of acoustic or elastic waves. In these materials, the dispersion properties and the energy transfer are controlled by selecting the geometry of the lattices and their constitutive material properties. Most designs, however, only affect one mode of energy propagation, transmitted either as acoustic airborne sound or as elastic structural vibrations. Here, we present a design methodology to attenuate both acoustic and elastic waves simultaneously in all polarizations. We experimentally realize a three-dimensional load-bearing architected lattice, composed of a single material, that responds in a broadband frequency range in all directions and polarizations for airborne sound and elastic vibrations simultaneously.
\end{abstract}

DOI: 10.1103/PhysRevApplied.10.054060

\section{INTRODUCTION}

Architected materials have the ability to influence the propagation of lattice vibrations or pressure waves across scales. These materials can attenuate elastic or acoustic energy by supporting the formation of forbidden frequency bands (band gaps) in their dispersion relation, where waves cannot propagate. These gaps form through two main mechanisms [1]: (1) Bragg scattering, where periodically repeated unit cells scatter waves with wavelength at the same order of the lattice spatial periodicity [2,3]; and (2) resonances, where locally resonating elements can attenuate waves with wavelength much larger than the lattice periodicity [4]. The resonances enable these lattices to retain properties that do not exist in conventional materials, such as negative effective mass or stiffness [5-7]. The existence of such band gaps within the frequency spectrum can be utilized for many applications, such as seismic protection [8,9], vibration or sound insulation [10-12], frequency filtering $[13,14]$, and wave guiding $[15,16]$, among others [17].

Architected lattices can be divided into two broad categories based on the host medium in which waves propagate $[5,6]$. (i) Acoustic lattices, controlling the propagation of pressure waves in fluids, such as air and water, usually feature rigid scatterers such as cylinders or spheres, capitalizing on destructive interference (Bragg-type scattering) [3,18-20]. Some designs also use resonances (and give rise to negative effective properties): for example, the inclusion

\footnotetext{
*bilal@caltech.edu
}

of heavy masses with rubber coatings that induce Mie-type resonances [4]. Other realizations include Helmholtz resonators featuring fluid chambers with single or multiple openings [21,22] or coiled space [23]. (ii) Elastic lattices, controlling the propagation of stress waves and vibrations in solid materials, can feature alternating material phases within the unit cell [2,24-28], with vast differences in mechanical properties, or single material with geometric features, such as holes $[29,30]$, leading to Bragg scattering. Another way to attenuate elastic waves is through resonating inclusions, such as pillars or heavy masses [31-33]. These realizations rely on strong resonance cutting through the dispersion curves to open subwavelength band gaps. Most recent studies on acoustic and elastic metamaterials have focused on the design and characterization of lattices with ever broader (and lower) frequency band gaps, in each separate domain of wave transport [6,33-42].

\section{DESIGN METHODOLOGY}

An architected lattice with the ability to attenuate both elastic and acoustic waves simultaneously in all directions remains elusive. Such a material can be useful for many applications; for example, in airplanes, ships, or automotive applications, where motors and/or fan units may produce both mechanical vibration and acoustic noise, compromising either operational comfort or functionality or both. In this work, we realize three-dimensional architected lattices that can simultaneously attenuate both acoustic (airborne sound) and elastic waves (vibrations) in all directions, over a broad range of frequencies [Figs. 1(a) and 1(b)]. Our design methodology capitalizes on both 
(a)

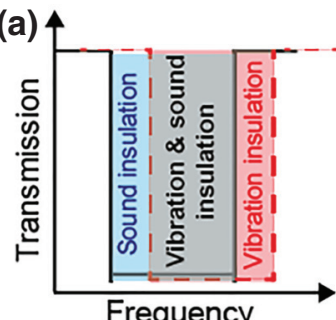

(b)

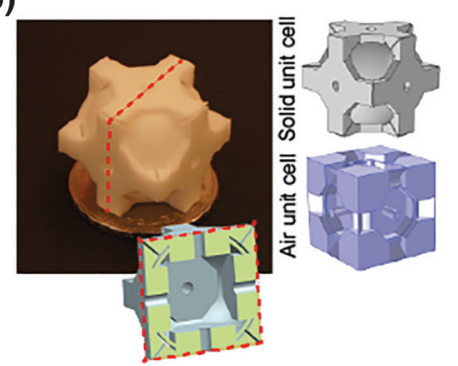

(c)

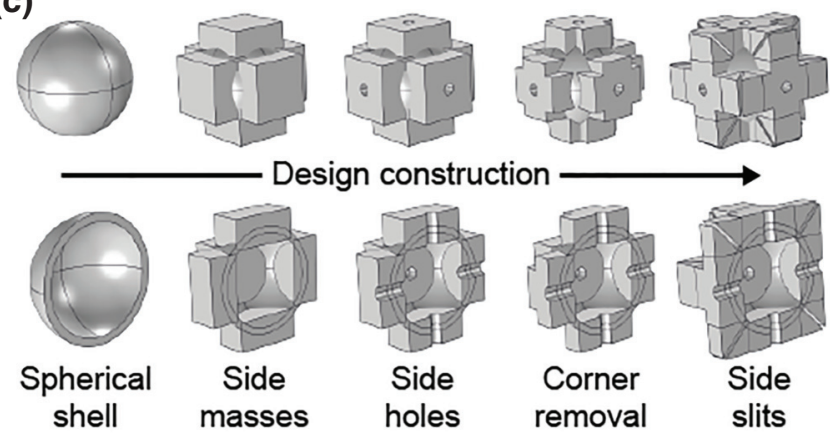

FIG. 1. (a) The conceptual frequency spectrum of a metamaterial with simultaneous band gaps for airborne sound and mechanical vibrations. (b) The basic building block for a material stopping both sound and vibrations in the same frequency range. Both the solid part and the air within the unit cell are plotted next to the physical prototype. (c) The design sequence starting from a hollow elastic sphere: top row, the design evolution of the 3D cell; bottom row, a view of the corresponding midsection plane cut-out.

scattering and resonances to open band gaps for sound and vibrations.

To construct our cubic unit cell, we start with an elastic spherical shell that works as an acoustic chamber resembling a Helmholtz resonator [Fig. 1(c)]. The shell also works as an elastic spring connecting six rectangular masses positioned at the center of each of the unit-cell faces. This spring-mass arrangement gives rise to Bragg scattering for elastic waves. For the chamber to function as a resonator for acoustic waves, we add a narrow cylindrical channel at each of the unit-cell faces. Afterward, we remove the corner of each face masses to add an extra acoustic chamber at each of the eight corners of the unit cell. Finally, we add four resonating "arms" to each of the six rectangular masses. The added arms function as locally resonating elements for elastic waves, while keeping each face separated from the neighboring faces. The arms also create a narrow slit connecting the corner chambers and introduce a second control over resonances for acoustic waves.

Based on this design methodology, the position of the band gaps for either sound or vibrations can be easily tuned; for example, by changing the narrow channel radius or the shell thickness. With this method, the attenuated bandwidth of sound frequencies can be chosen independently from the attenuated vibration frequency ranges. In other words, one can create multiple band gaps in the audible regime for sound waves and have other band gaps in similar (or different) frequencies for elastic vibrations. The realized lattice is load bearing (see the Supplemental Material) and the underlying principle of wave attenuation is scale and material agnostic.

To investigate the validity of our approach, we first consider an infinite medium model, where a single unit cell is analyzed using Bloch periodic boundary conditions [43]. We assume small deformations and therefore neglect acoustoelastic coupling. The dispersion curves of the unit cells are calculated using the wave equations for heterogeneous media [44] within an infinite medium. We solve both the acoustic and the elastic equations using the finiteelement method (COMSOL 5.2). The solution is the wave function $u(x, \kappa ; t)=\tilde{u}(x) \exp \left[i\left(\kappa^{\top} x-\omega t\right)\right]$, where $\tilde{u}$ is the Bloch displacement vector, $x$ is the position vector, $\kappa$ is the wavenumber, $\omega$ is the frequency, and $t$ is time. The dispersion curves relating the wavenumber to the frequency, in nondimensional units, show band gaps (gray shaded regions) for both elastic [Fig. 2(a)] and acoustic waves [Fig. 2(b)]. The dispersion curves are normalized by multiplying the operational frequency by the unit-cell size divided by the speed of the wave in the medium, $\Omega=f a / c$.
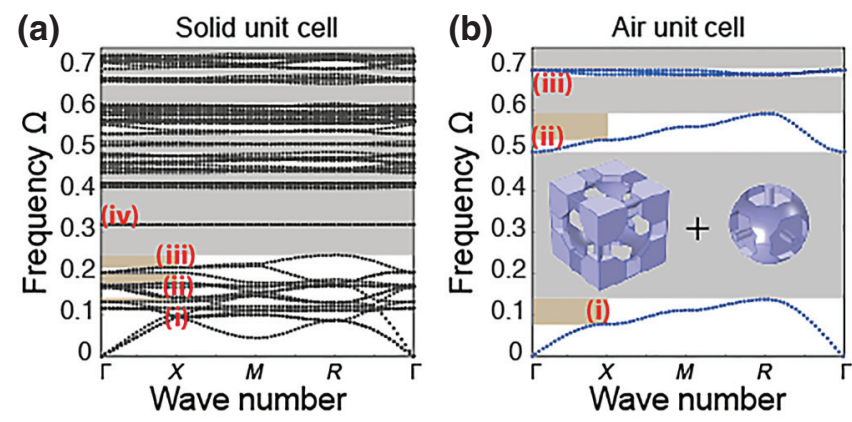

(c)
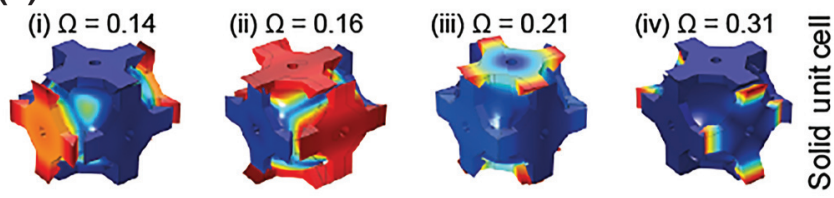

(d)


FIG. 2. The dispersion curves of the metamaterial for (a) mechanical vibrations and (b) airborne sound: full band gaps are highlighted in gray and partial ones are in brown. (c) Selected elastic mode shapes of the solid unit cell. (d) Selected acoustic mode shapes of the air unit cell. 
It should be noted that while the unit-cell size is the same in both elastic and acoustic cases, the wave speeds are not. Therefore, having a band gap in both plots - at $\Omega=0.3$, for instance - does not necessarily guarantee a simultaneous band gap in the dimensional frequency domain.

\section{RESULTS}

To visualize the vibrational mode shapes of the solid unit cell, we superimpose the displacement profiles as a heat map over its geometry for four different frequencies in Fig. 2(c). The mode shapes resemble (i) longitudinal, (ii) shear, and (iii) rotational modes of the face masses in the unit cell. We also plot (iv) a resonant mode shape of the arms, which manifests itself within the first full band gap in the frequency spectrum at $\Omega=0.31$. The acoustic pressure profiles of the air unit cell are superimposed as a heat map over its geometry for four different frequencies in Fig. 2(d). The mode shapes show the resonance mode of the spherical chamber [(i) and (iii)] and the corner chambers [(ii) and (iv)].

As a proof-of-concept demonstration, we first realize an array of seven unit cells tessellated along one direction [Figs. 3(a) and 3(d)]. We fabricate our samples by additive manufacturing (laser sintering) using polyamide-12 (PA-12) polymer (the measured Young's modulus and density are $E=1.6 \mathrm{GPa}$ and $\rho=1200 \mathrm{Kg} / \mathrm{m}^{3}$ ). The lattice spacing is $a=34 \mathrm{~mm}$. The elastic response of the metamaterial is characterized by harmonically exciting one of its ends with a mechanical shaker (Brüel \& Kjaer Type 4810) and measuring the transmitted vibrations with a laser Doppler vibrometer (LDV) (Polytec OFV-505 with a OFV-5000 decoder, using a VD-06 decoder card) at its other end [Fig. 5(a)]. We sweep through frequencies ranging from 1 to $16 \mathrm{kHz}$ and record the amplitude of the transmitted vibrations [Fig. 3(b)]. We replicate the experiment numerically using the finite-element method, by applying a harmonic load along the $x$ direction and recording the amplitude of the displacement at the opposite end of the structure. The theoretically predicted band gaps are highlighted in gray in [Fig. 3(b)]. The numerically computed displacements are superimposed as a heat map over the structure for six different frequencies within both pass (top) and stop (bottom) bands in Fig. 3(c). The experiments and the numerical results agree well. We note the existence of low-amplitude regions within the transmission plot that do not coincide with a band gap. They correspond to a pass band with rotational or shear polarizations and are not excited longitudinally, in either experiments or simulations.

To test the acoustic response of the metamaterial, we enclose the sample within a custom-made impedance tube [Fig. 3(d)] inside an acoustic chamber. Chirp signals are generated with a loudspeaker (model Clarion (a)

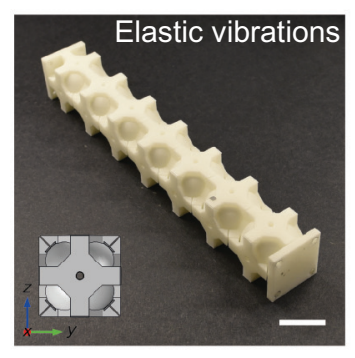

(d)

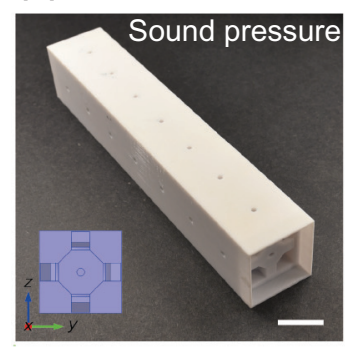

(b)

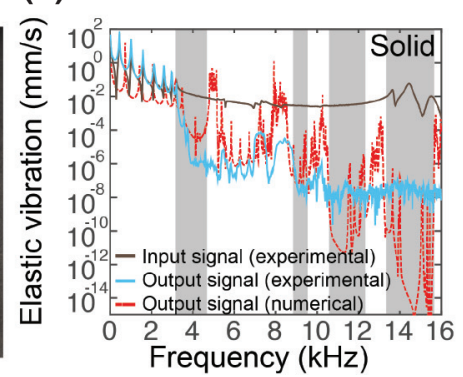

(e)



(c)

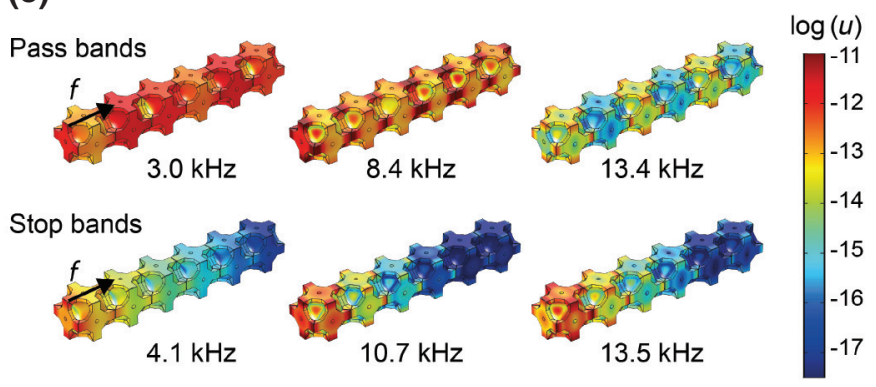

(f)

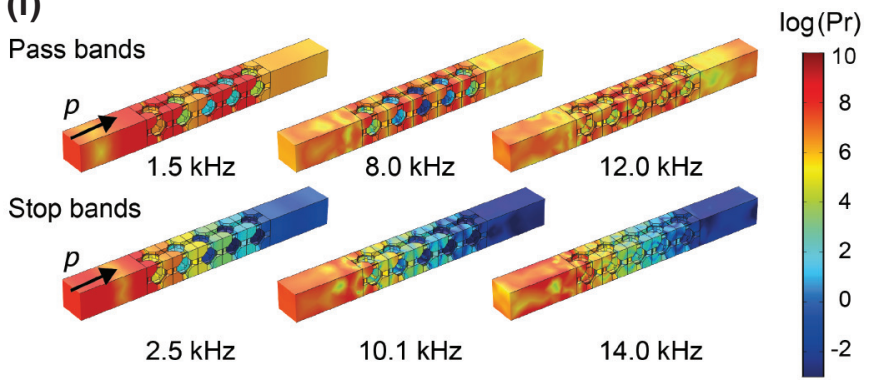

FIG. 3. (a) The metamaterial used for the elastic vibration experiment. (b) The numerical and experimental frequency response functions for elastic vibrations. The gray shaded areas represent the location of the band gaps calculated with Bloch analysis. (c) Selected mode shapes of the metamaterial at pass bands and stop bands for elastic waves. (d) The same metamaterial enclosed in a tube for sound transmission experiments. (e) The numerical and experimental frequency response functions. (f) Selected mode shapes of the metamaterial within pass and stop bands for sound waves. The scale bars in (a) and (d) are $25 \mathrm{~mm}$. The color bars in (c) and (f) represent the logarithms of displacement and pressure, respectively. 
SRE212H) on one end of the tube. Two microphones (G.R.A.S. 40BD) are used to record the generated and transmitted signal, on each side of the metamaterial [Fig. 5(b)]. We experimentally observe more than $35 \mathrm{~dB}$ reduction in the transmitted sound along the propagation direction. We model the experiment numerically, considering the tube as a rigid boundary and reproducing the geometry of the sample using the finite-element method. We generate the excitation as a point source on one of the ends of the tube and plot the intensity of the pressure field on the other end [Fig. 3(e)]. As for mechanical vibrations, the band gaps calculated with Bloch analysis are highlighted in gray. The numerically computed pressure fields are plotted as heat maps for six different frequencies within both pass (top) and stop (bottom) bands in Fig. 3(f). A good agreement between theory, numerical simulations, and experiments is observed. The results demonstrate the ability of our metamaterial to simultaneously attenuate both airborne sound and mechanical vibrations, in selected frequency ranges. It should be noted that the presence of simultaneous band gaps for elastic vibrations and airborne sound is not automatically granted and has to be designed for. For instance, at $2 \mathrm{kHz}$, the metamaterial can attenuate sound but not vibrations. The opposite is true at $12 \mathrm{kHz}$, where the metamaterial can shield elastic vibrations but not airborne sound.

To study the effect of structural geometry on the sound attenuation properties, we perform numerical simulations comparing the response of a solid slab, a slab with a thin air channel, and our structured material, all made of polyamide-12 (Fig. 6). We observe that the structured materials, with a density 6 times lower than the solid slab, outperforms the solid barrier by up to $30 \%$ within the band-gap frequency range. It is worth noting that our metamaterial is porous. Adding an air opening within the reference solid material dramatically reduces its soundshielding effectiveness due to impedance matching with surrounding air. The attenuation level between the solid sample (with an air opening) and our structured material is almost half.

The positions of the band gaps within the frequency spectrum, for either sound or vibrations, can be altered by different means. For example, changing the lattice constant would change the band-gap position for both sound and vibrations. The use of a different constitutive material-for example, with a higher Young's modulus - would shift the elastic band gaps to higher frequencies, while keeping the acoustic gaps unchanged. However, even while keeping the same material and lattice constant, our design principle allows for decoupling the position of the band gaps for sound and vibrations. For example, consider a unit cell fabricated with polyamide- 12 polymer and a lattice spacing of $25 \mathrm{~mm}$. Changing the radius of the hole at the center of the unit cell [Fig. 7(a)], from 0.5 to $1.75 \mathrm{~mm}$ can change the lower edge of the first complete acoustic band gap from 500 to $2500 \mathrm{~Hz}$ [Fig. 7(b)], with negligible effects on the elastic waves traveling through the media. Changing the thickness of the shells enclosing the air chamber in each (a)

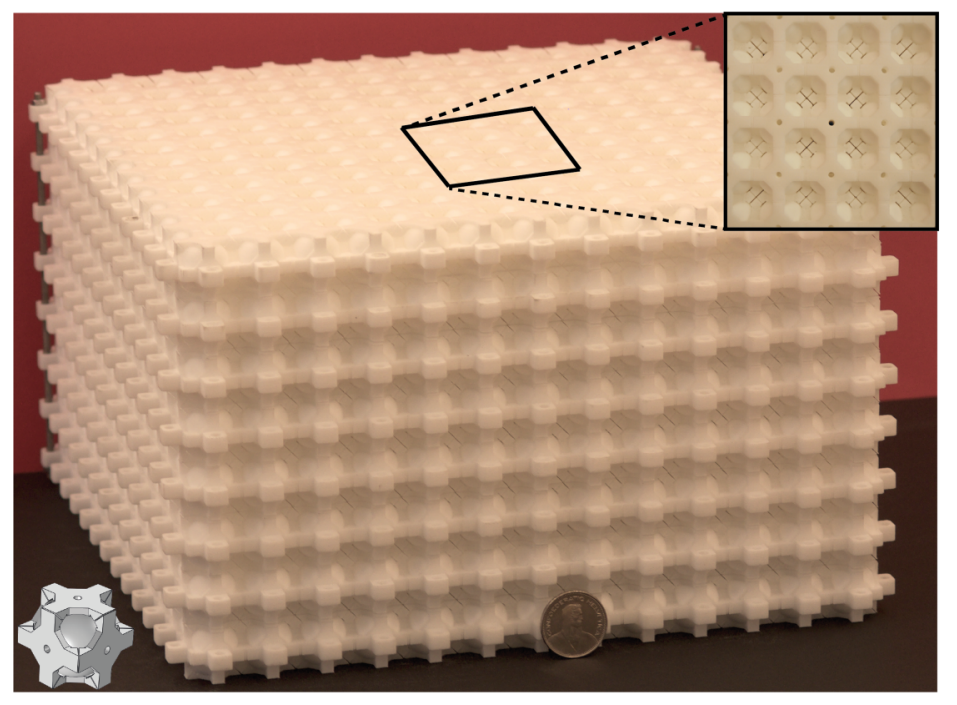

(b)

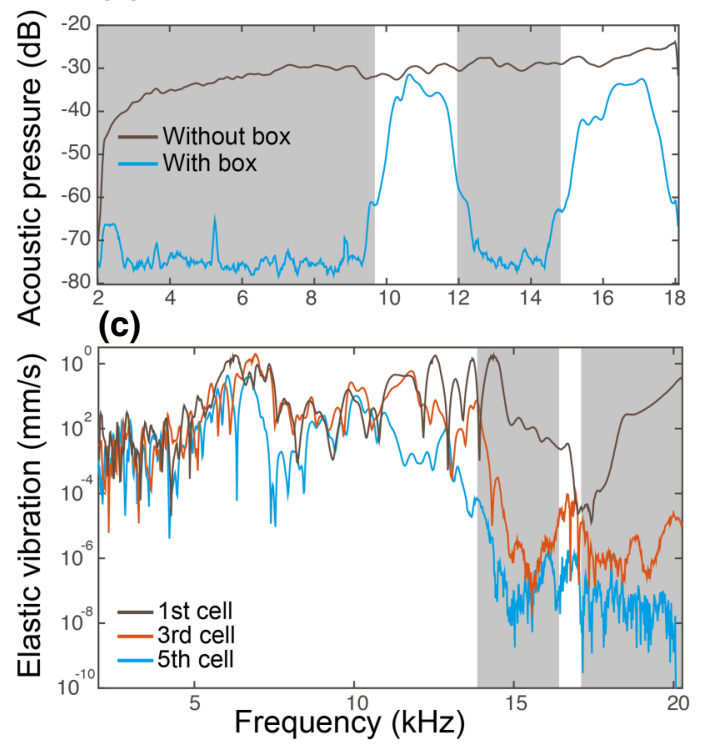

FIG. 4. (a) A three-dimensional realization of the metamaterial consisting of $13 \times 13 \times 8$ unit cells with a lattice constant $a=25$ $\mathrm{mm}$. The material box encloses a piezoelectric transducer for generating mechanical vibrations and a loudspeaker for airborne sound. (b) The acoustic frequency response of the metamaterial using a microphone $8 \mathrm{~cm}$ above the box compared to the transmission of same speaker and microphone without our material. (c) The elastic frequency response of the metamaterial using LDV at different distances from the mechanical wave source (one, three, and five unit cells). 
unit cell [Fig. 7(c)], from 0.3 to $0.9 \mathrm{~mm}$, can shift the lower edge of the elastic band gap from 10 to $15 \mathrm{kHz}$ [Fig. 7(d)]. The change in shell thickness has a negligible effect on the acoustic response of the metamaterial. Similarly, changing the side openings or the shape and/or mass of the outer face of the unit cell would significantly change either the acoustic or the elastic response of the metamaterial, respectively, without significantly affecting the other.

To demonstrate the effectiveness of the design methodology in attenuating both sound and vibrations in all directions, we realize a $13 \times 13 \times 8$ lattice with $a=25 \mathrm{~mm}$ [Fig. 4(a)]. The fabricated box has a cavity of $3 \times 3 \times 3$ unit cells in its bottom center to host a mechanical transducer and a loudspeaker for exciting both structural and sound waves, respectively. Therefore, the effective number of unit cells in any direction $( \pm x, \pm y,+z)$ is five. To test the acoustic insulation, we embed a loudspeaker (model Clarion SRE212H) inside the metamaterial and measure the amplitude of the transmitted sound through the lattice with a $1 / 4$-inch $(6.35-\mathrm{mm})$ microphone (G.R.A.S. 40BD). The test is carried out in an insulated acoustic chamber, moving the microphone in different locations around the metamaterial (see the Supplemental Material). We compare the signal propagating through the metamaterials to sound waves recorded without the lattice at the same distance from the source. The measured band gaps span frequencies from 2 to $9.5 \mathrm{kHz}$ and from 12 to $14.8 \mathrm{kHz}$. The attenuated frequency ranges translate to about $60 \%$ of the entire audible range. With the metamaterial, we measure more than $35 \mathrm{~dB}$ attenuation of the sound-wave amplitude in all directions. To test the insulation from mechanical vibrations, we embed a piezoelectric transducer (Piezo Systems $25 \times 25 \times 2 \mathrm{~mm}^{3}$ ) [45] within the metamaterial cavity and induce harmonic excitations at different frequencies. We measure the transmitted vibrations through the material on its outer surface at distances of one, three, and five unit cells from the vibration source using the LDV. At the targeted frequency range (highlighted in gray), we observe a significant reduction in the measured wave velocities after the third unit cell.

\section{CONCLUSION}

Our design methodology allows for the independent tuning of band-gap frequency ranges for each domain (i.e., either elastic vibrations or airborne sound) in a load-bearing metamaterial. Our findings could open new opportunities for the design of advanced multifunctional metamaterials, for application in transportation vessels, machinery, and building acoustics.

\section{APPENDIX}

\section{Characterization of one-dimensional metamaterial}

To test our metamaterial properties, we fabricate two arrays composed of seven unit cells. For elastic vibration testing, we add two thin plates $(5 \mathrm{~mm})$ at each end of the metamaterial array. We mount an electromechanical shaker against one of the plates and measure the transmitted signal at the second plate [Fig. 5(a)]. We cover the free end (i.e., the second plate) with a reflective tape and record its movement (displacement and velocity) using a laser Doppler vibrometer. The experimental setup guarantees complete isolation of the metamaterial sample from any undesired vibrations through the table. The excitation signal is sent to the electromechanical shaker from the PC through an audio amplifier (Topping TP22). The measured velocities are sent back to the PC through a lock-in amplifier model (Zürich Instruments HF2LI).

For airborne acoustic testing, we fabricate the metamaterial enclosed in a tube with a square cross section. The printed tube has circular holes aligned with those in the metamaterial sample, to ease the removal of excess powder from the printing process. The side holes are then soundproofed using Blu-tack, to prevent any sound leak from the metamaterial to the chamber and to ensure full transmission of the wave through the longitudinal direction of the metamaterial. We fit both ends of the metamaterial in a custom impedance tube with a square profile. The tube has two microphones, mounted at a distance of $70 \mathrm{~mm}$ from the edges of the metamaterial sample. A loudspeaker is mounted at one end of the tube, while the other end is fitted within the padding of the acoustic chamber [Fig. 5(b)].

As a control, we simulate the sound-attenuation characteristics of an open air channel, a solid block made of PA-12, and a PA-12 block with a small open cylindrical channel $($ diameter $=5 \mathrm{~mm}$ ). We use the COMSOL multiphysics acoustics module to simulate the sound-pressure fields. An impedance tube identical to the custom-made (a)

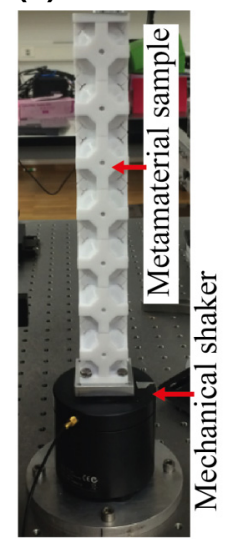

Elastic vibrations (b)

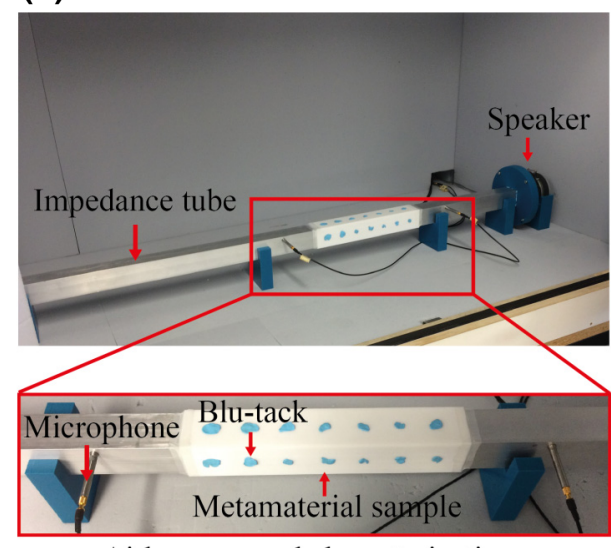

Airborne sound characterization
FIG. 5. One-dimensional metamaterial characterization: (a) the mechanical vibration excitation of a $7 \times 1$ metamaterial sample using a mechanical shaker; (b) the airborne sound excitation of a $7 \times 1$ metamaterial sample using a loudspeaker and a custommade impedance tube with a square cross section. 
(a)
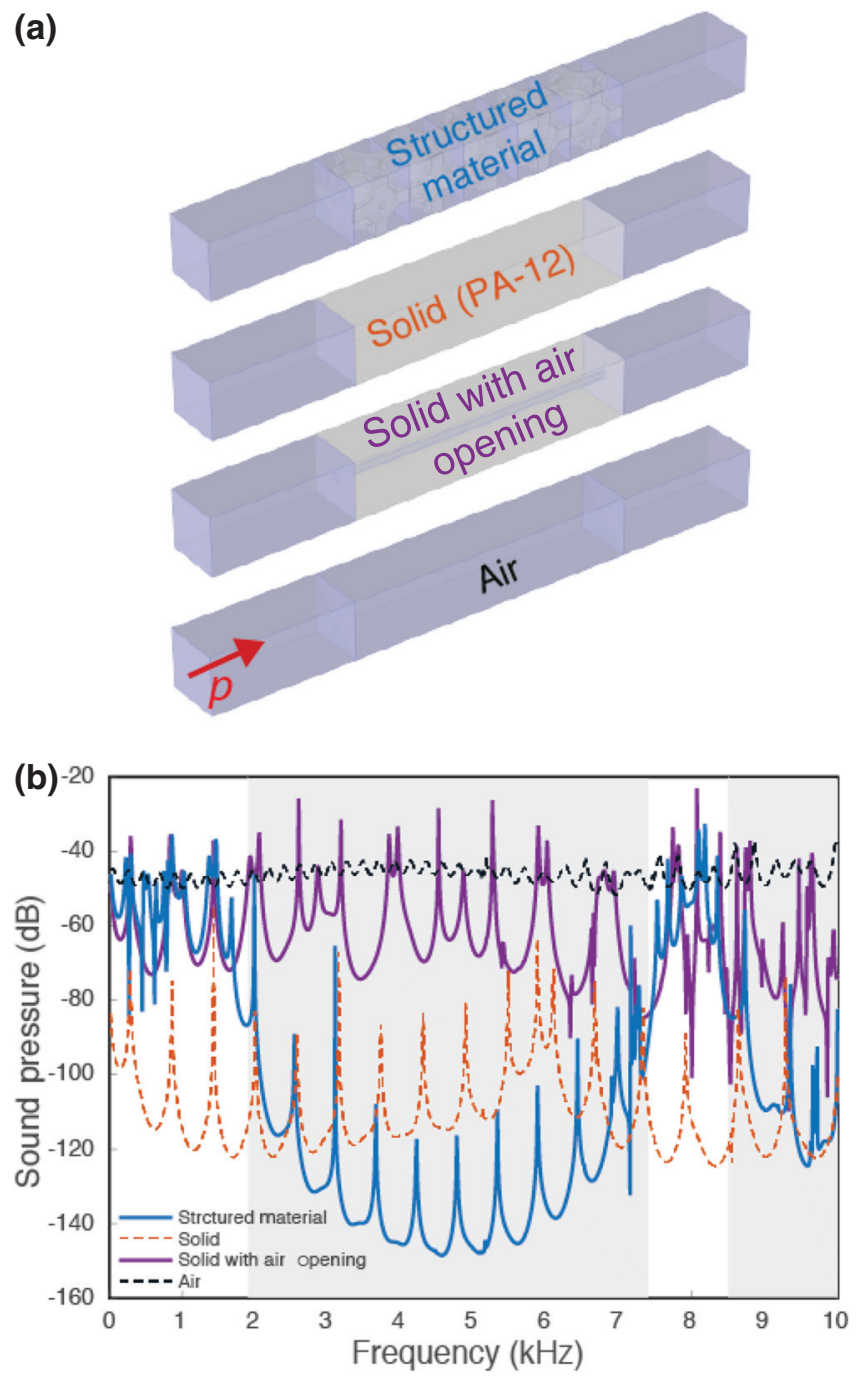

FIG. 6. A comparison of the acoustic attenuation of different materials. (a) A schematic of the tested materials: (i) a structured material with air openings, (ii) an airtight solid block made of PA-12, (iii) a PA-12 block with a cylindrical opening with a diameter of $5 \mathrm{~mm}$, and (iv) an open air channel as a reference. (b) The numerical frequency response function (FRF) of the sound-pressure field at the end of the impedance tube.

one in Fig. 5(b) is modeled in the numerical simulations. The pressure wave is introduced at one end of the tube as a point source and the resulting pressure field is measured at the other end of the sample. We compare the performance of the three samples against our structured metamaterial [Fig. 6(a)]. The open air channel has no attenuation capabilities, as expected; therefore, it sets the bar for the sound-pressure level. Due to impedance mismatch $\left(Z_{\text {air }}=\right.$ $\left.0.000445 \mathrm{~kg} / \mathrm{m}^{2} \mathrm{~s} \times 10^{6}, Z_{\mathrm{PA}-12}=1.5 \mathrm{~kg} / \mathrm{m}^{2} \mathrm{~s} \times 10^{6}\right)$, an airtight block of PA-12 attenuates a large amount of the incident sound energy; however, the effectiveness of such an approach degrades exponentially with a small air opening. Such a scenario is common with the assembly of (a)

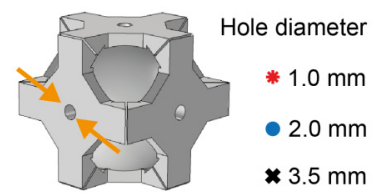

(c)

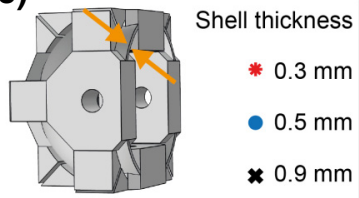

(b)



(d)

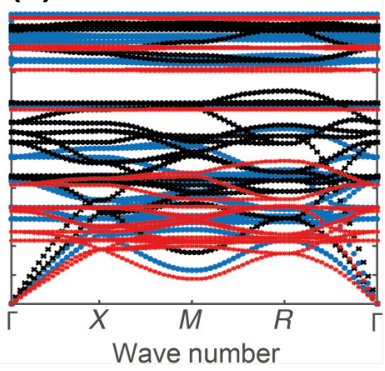

FIG. 7. The control parameters for changing the position of the band gap for (a),(b) acoustic or (c),(d) elastic waves independently. (a) Varying the hole diameter changes the channel width for sound waves and therefore their characteristic acoustic dispersion. (b) Acoustic wave dispersion curves for three different diameters: 1, 2, and $3.5 \mathrm{~mm}$. (c) Varying the shell thickness changes the effective coupling between unit-cell parts for elastic waves and therefore their characteristic elastic dispersion. (d) Elastic-wave dispersion curves for three different shell thicknesses: $0.3,0.5$, and $0.9 \mathrm{~mm}$.

parts, mechanisms, or when open air systems are required (e.g., for cooling purposes). In the case of our structured materials, the attenuation level is equivalent to the homogeneous material with an open channel in the pass-band frequencies. Within the frequency range of the band gap, the structured material has a superior attenuation profile for sound waves compared to the three other modeled samples.

\section{Fabrication of three-dimensional metamaterial}

To characterize the metamaterial in all directions, we fabricate a "box" consisting of $13 \times 13 \times 8$ unit cells each measuring $25 \mathrm{~mm}$. To speed up the printing process, simplify the removal of the excess printing powder, and ease the mounting of vibrations and noise sources, we print each of the box layers separately. Five of the printed layers are composed of $13 \times 13$ unit cells, while the remaining three layers have a void with an equivalent space of $3 \times 3$ unit cells. The void hosts both a loudspeaker and a piezoelectric transducer. To ensure the alignment of the unit cells in the printed layers, we incorporate 13 holes at each side of the printed layers. Following the assembly of the layers, a long screw passes vertically through the holes and is secured with two bolts at each of its ends [Fig. 8(a)].

\section{Characterization of three-dimensional metamaterial}

To test the response of the metamaterial to airborne sound, we excite the box from within, using a loudspeaker 
(a)



Box bottom

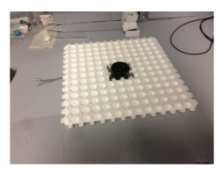

First layer

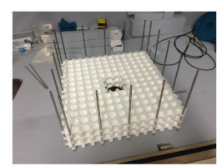

First three layers (b)

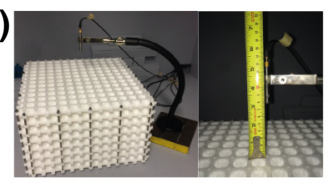

Top measurement

(c)

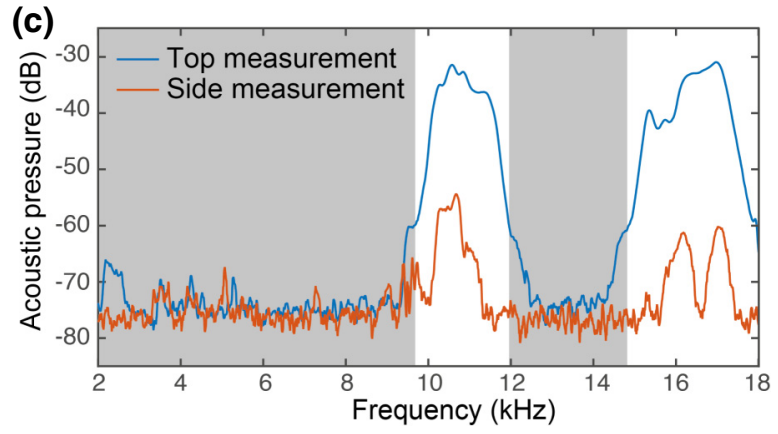

FIG. 8. (a) A metamaterial assembly of $13 \times 13 \times 3$ unit cells with a speaker attached to the bottom layer as a source for airborne sound. (b) A fully assembled metamaterial box with microphones positioned facing the speaker (left) and to its side (right). (c) The measured acoustic pressure through the metamaterial acquired through forward-facing and side-facing microphones.

[Fig. 8(a)]. Since the box is three-dimensional, there is no need to cover the pores of the metamaterial. The box is tested within the same acoustic chamber to ensure insulation from surrounding acoustic noise. We place the microphone at each one of the sides of the metamaterial box to capture the acoustic radiation in all directions [Fig. 8(b)]. Both the side and top measurements confirm the existence of the band gaps predicted from the unit-cell analysis [gray regions in Fig. 8(c)]. It should be noted, however, that the transmission in the side measurements has generally lower amplitude than the top one, as the speaker is facing upward.

To test the response of the metamaterial to elastic vibrations, we excite the box from within using a piezoelectric plate (Piezo Systems $25 \times 25 \times 2 \mathrm{~mm}^{3}$ ) [45]. The transmitted vibrations are then measured using the laser Doppler vibrometer at various points within the metamaterial. The measurements taken for a single sheet (2D) agree well with the full box measurement [Fig. 9(b)].

\section{The load-bearing capacity of the metamaterial}

We characterize the effective static stiffness of the metamaterial by comparing a block of PA-12 against a single unit cell in a compression test using an Instron 3000 machine. As expected, the thin features of the unit cell cause a reduction in stiffness of the metamaterial by about (a)

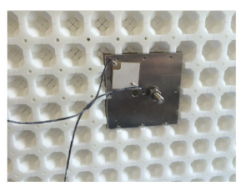

Box bottom



First layer

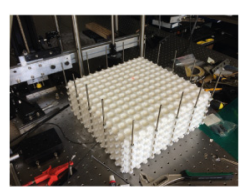

First five layers

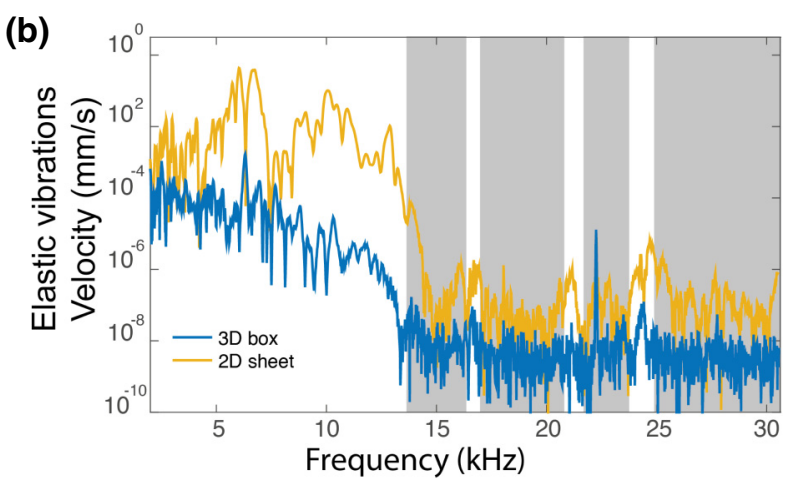

FIG. 9. (a) A metamaterial assembly of $13 \times 13 \times 5$ unit cells with a piezoelectric plate attached to the bottom layer as a source for mechanical vibrations. (b) The measured velocity transmitted through one sheet of the metamaterial at the fifth unit cell and the measured response after assembling five layers of metamaterials in the vertical direction.

an order of magnitude with reference to the bulk material. A complementary numerical simulation of the compression test shows the stress concentration within the unit cell (inset in Fig. 10). We use the COMSOL structure mechanics module to perform the numerical test. We add a prescribed displacement as a boundary condition on the top surface of the unit cell in the $z$ direction, while keeping the bottom face of the unit cell fixed. The numerical results suggest that the stiffness of the metamaterial can be greatly increased by increasing the thickness of the connecting (a)

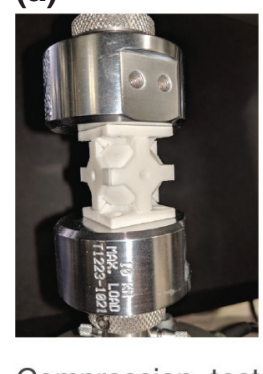

Compression test

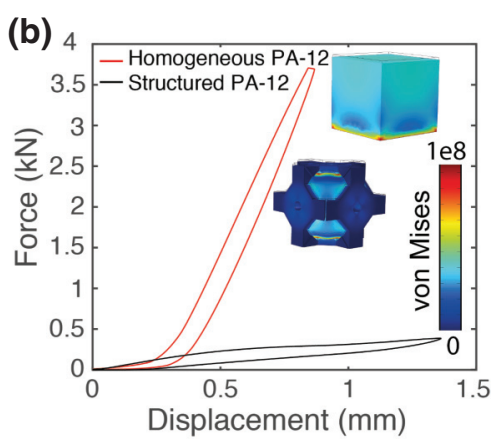

FIG. 10. The experimental characterization of the load-bearing capabilities of the structured metamaterial in comparison to a homogeneous cube of the same material (PA-12). The inset shows the numerical calculation of the von Mises stress for a homogeneous and structured cube under a compression load with $1 \mathrm{~mm}$ strain. 
shells within the unit cell. It is worth noting that the metamaterial is lighter than the bulk PA-12 by a factor of 6 , due to material removal.

[1] Pierre A. Deymier, Acoustic Metamaterials and Phononic Crystals (Springer Science \& Business Media, Berlin, 2013), Vol. 173.

[2] M. S. Kushwaha, P. Halevi, L. Dobrzynski, and B. DjafariRouhani, Acoustic Band Structure of Periodic Elastic Composites, Phys. Rev. Lett. 71, 2022 (1993).

[3] M. Sigalas and E. N. Economou, Band structure of elastic waves in two dimensional systems, Solid State Commun. 86, 141 (1993).

[4] Zhengyou Liu, Xixiang Zhang, Yiwei Mao, Y. Y. Zhu, Zhiyu Yang, C. T. Chan, and Ping Sheng, Locally resonant sonic materials, Science 289, 1734 (2000).

[5] Johan Christensen, Muamer Kadic, Oliver Kraft, and Martin Wegener, Vibrant times for mechanical metamaterials, MRS Commun. 5, 453 (2015).

[6] Steven A. Cummer, Johan Christensen, and Andrea Alù, Controlling sound with acoustic metamaterials, Nat. Rev. Mater. 1, 16001 (2016).

[7] Guancong Ma and Ping Sheng, Acoustic metamaterials: From local resonances to broad horizons, Sci. Adv. 2, e1501595 (2016).

[8] Sang-Hoon Kim and Mukunda P. Das, Seismic waveguide of metamaterials, Mod. Phys. Lett. B 26, 1250105 (2012).

[9] Stéphane Brûlé, E. H. Javelaud, Stefan Enoch, and Sébastien Guenneau, Experiments on Seismic Metamaterials: Molding Surface Waves, Phys. Rev. Lett. 112, 133901 (2014).

[10] Z. Yang, H. M. Dai, N. H. Chan, G. C. Ma, and Ping Sheng, Acoustic metamaterial panels for sound attenuation in the 50-1000 Hz regime, Appl. Phys. Lett. 96, 041906 (2010).

[11] Jun Mei, Guancong Ma, Min Yang, Zhiyu Yang, Weijia Wen, and Ping Sheng, Dark acoustic metamaterials as super absorbers for low-frequency sound, Nat. Commun. 3, 756 (2012).

[12] Fuyin Ma, Jiu Hui Wu, Meng Huang, Weiquan Zhang, and Siwen Zhang, A purely flexible lightweight membrane-type acoustic metamaterial, J. Phys. D Appl. Phys. 48, 175105 (2015).

[13] Yan Pennec, B. Djafari-Rouhani, J. O. Vasseur, Abdelkrim Khelif, and P. A. Deymier, Tunable filtering and demultiplexing in phononic crystals with hollow cylinders, Phys. Rev. E 69, 046608 (2004).

[14] Cory J. Rupp, Martin L. Dunn, and Kurt Maute, Switchable phononic wave filtering, guiding, harvesting, and actuating in polarization-patterned piezoelectric solids, Appl. Phys. Lett. 96, 111902 (2010).

[15] M. Torres, F. R. Montero deEspinosa, D. Garcia-Pablos, and N. Garcia, Sonic Band Gaps in Finite Elastic Media: Surface States and Localization Phenomena in Linear and Point Defects, Phys. Rev. Lett. 82, 3054 (1999).

[16] Cory J. Rupp, Anton Evgrafov, Kurt Maute, and Martin L. Dunn, Design of phononic materials/structures for surface wave devices using topology optimization, Struct. Multidiscipl. Optim. 34, 111 (2007).
[17] Martin Maldovan, Sound and heat revolutions in phononics, Nature 503, 209 (2013).

[18] M. S. Kushwaha and P. Halevi, Giant acoustic stop bands in two-dimensional periodic arrays of liquid cylinders, Appl. Phys. Lett. 69, 31 (1996).

[19] Manvir S. Kushwaha and P. Halevi, Stop bands for cubic arrays of spherical balloons, J. Acoust. Soc. Am. 101, 619 (1997).

[20] Juan V. Sánchez-Pérez, D. Caballero, Rosa MártinezSala, Constanza Rubio, José Sánchez-Dehesa, Francisco Meseguer, Jaime Llinares, and F. Gálvez, Sound Attenuation by a Two-Dimensional Array of Rigid Cylinders, Phys. Rev. Lett. 80, 5325 (1998).

[21] Nicholas Fang, Dongjuan Xi, Jianyi Xu, Muralidhar Ambati, Werayut Srituravanich, Cheng Sun, and Xiang Zhang, Ultrasonic metamaterials with negative modulus, Nat. Mater. 5, 452 (2006).

[22] Sam Hyeon Lee, Choon Mahn Park, Yong Mun Seo, Zhi Guo Wang, and Chul Koo Kim, Acoustic metamaterial with negative modulus, J. Phys. Condens. Matter 21, 175704 (2009).

[23] Yangbo Xie, Bogdan-Ioan Popa, Lucian Zigoneanu, and Steven A. Cummer, Measurement of a Broadband Negative Index with Space-Coiling Acoustic Metamaterials, Phys. Rev. Lett. 110, 175501 (2013).

[24] Michael M. Sigalas and Eleftherios N. Economou, Elastic and acoustic wave band structure, J. Sound Vib. 158, 377 (1992).

[25] Manvir S. Kushwaha, P. Halevi, G. Martinez, Leonard Dobrzynski, and Bahram Djafari-Rouhani, Theory of acoustic band structure of periodic elastic composites, Phys. Rev. B 49, 2313 (1994).

[26] E. N. Economou and M. Sigalas, Stop bands for elastic waves in periodic composite materials, J. Acoust. Soc. Am. 95, 1734 (1994).

[27] J. O. Vasseur, B. Djafari-Rouhani, L. Dobrzynski, M. S. Kushwaha, and P. Halevi, Complete acoustic band gaps in periodic fibre reinforced composite materials: The carbon/epoxy composite and some metallic systems, J. Phys. Condens. Matter 6, 8759 (1994).

[28] F. R. Montero De Espinosa, E. Jimenez, and M. Torres, Ultrasonic Band Gap in a Periodic Two-Dimensional Composite, Phys. Rev. Lett. 80, 1208 (1998).

[29] Martin Maldovan and Edwin L. Thomas, Periodic Materials and Interference Lithography: For Photonics, Phononics and Mechanics (John Wiley \& Sons, Weinheim, 2009).

[30] O. R. Bilal and M. I. Hussein, Ultrawide phononic band gap for combined in-plane and out-of-plane waves, Phys. Rev. E 84, 065701 (2011).

[31] Y. Pennec, B. Djafari-Rouhani, H. Larabi, J. O. Vasseur, and A. C. Hladky-Hennion, Low-frequency gaps in a phononic crystal constituted of cylindrical dots deposited on a thin homogeneous plate, Phys. Rev. B 78, 104105 (2008).

[32] Tsung-Tsong Wu, Zi-Gui Huang, Tzu-Chin Tsai, and Tzung-Chen Wu, Evidence of complete band gap and resonances in a plate with periodic stubbed surface, Appl. Phys. Lett. 93, 111902 (2008).

[33] Kathryn H. Matlack, Anton Bauhofer, Sebastian Krödel, Antonio Palermo, and Chiara Daraio, Composite 3D-printed metastructures for low-frequency and broadband 
vibration absorption, Proc. Natl Acad. Sci. 113, 8386 (2016).

[34] Mahmoud I. Hussein, Michael J. Leamy, and Massimo Ruzzene, Dynamics of phononic materials and structures: Historical origins, recent progress, and future outlook, Appl. Mech. Rev. 66, 040802 (2014).

[35] L. D'Alessandro, E. Belloni, R. Ardito, A. Corigliano, and F. Braghin, Modeling and experimental verification of an ultra-wide band gap in 3D phononic crystal, Appl. Phys. Lett. 109, 221907 (2016).

[36] Frieder Lucklum and Michael J. Vellekoop, Bandgap engineering of three-dimensional phononic crystals in a simple cubic lattice, Appl. Phys. Lett. 113, 201902 (2018).

[37] Franziska Warmuth, Maximilian Wormser, and Carolin Körner, Single phase 3D phononic band gap material, Sci. Rep. 7, 3843 (2017).

[38] Luca D'Alessandro, Valentina Zega, Raffaele Ardito, and Alberto Corigliano, 3D auxetic single material periodic structure with ultra-wide tunable bandgap, Sci. Rep. 8, 2262 (2018).
[39] S. Taniker and C. Yilmaz, Design, analysis and experimental investigation of three-dimensional structures with inertial amplification induced vibration stop bands, Int. J. Solids Struct. 72, 88 (2015).

[40] Maximilian Wormser, Fabian Wein, Michael Stingl, and Carolin Körner, Design and additive manufacturing of 3D phononic band gap structures based on gradient based optimization, Materials 10, 1125 (2017).

[41] A. Foehr, O. R. Bilal, S. D. Huber, and C. Daraio, Spiral-based Phononic Plates: From Wave Beaming to Topological Insulators, Phys. Rev. Lett. 120, 205501 (2018).

[42] Osama R. Bilal, André Foehr, and Chiara Daraio, Observation of trampoline phenomena in 3D-printed metamaterial plates, Extreme Mech. Lett. 15, 103 (2017).

[43] Felix Bloch, About the quantum mechanics of electrons in crystal lattices, J. Phys. 52, 555 (1929).

[44] Karl F. Graff, Wave Motion in Elastic Solids (Courier Corporation, New York, 2012).

[45] http://www.Piezo.com 